\title{
中缝背核5-埥色胺能神经元与阿片 受体关系的形态研究
}

\author{
马青平 邓云平1）艾民康 ${ }^{1}$ 韩济生 \\ (北京医科大学神经科学研究中心,北京 $100083 ;^{2}$ ) 同济医科大学针刺镇㮤原理研究室武汉, 4300340)
}

\section{关链调中继背核、5-羟色胺、阿片受体、免疫细胞化学、放射自显影}

许多资料表明, 中㖓背核是 5-羟色胺 (5-HT) 能神经元大量存在的部位 ${ }^{[1]}$, 同时也是阿 片受体比较密集的区域, 由此处发出的 5-HT 能纤维上行到达间脑和端脑的若干核团山. 中 㖓背核在脑内镇痛系统中占有重要地位: 电刺敫中琏背核能产生明显的镇痛作用 ${ }^{[3]}$, 中脑导水 管周围灰质 (PAG)内注人微量吗啡引起的镇痛很可能是通过激活中㖓背核的 5-HT 能神经 元而发挥作用的 ${ }^{6}$. 由于 5-HT 能神经元和阿片受体的形态定位关系尚不清楚, 吗啡激活 5HT 能神经元的形态机制一直没有阐明. 本工作应用免疫细胞化学与阿片受体放射自显影双 重显示技术,对中琏 5-HT 能神经元与阿片受体的形态定位关系进行了研究。

\section{一、材料与方法}

大鼠经腹腔注射 $10 \%$ 水合氯醛 $(300 \mathrm{mg} / \mathrm{kg}$ 体重)麻醉后, 经主动脉先灌人生理盐水 100 $\mathrm{ml}$, 随即灌人冷 $1 \%$ 多聚甲醛溶液 (0.1 mol/L 磷酸盐缓冲液配 制, $\mathrm{pH} 7.4) 400 \mathrm{ml}, 30 \mathrm{~min}$ 灌完. 取脑, 投人相同固定液 $4^{\circ} \mathrm{C}$ 固定 $12 \mathrm{~h}$ 左右后, 移人 $20 \%$ 蔗糖溶液 $(0.1 \mathrm{~mol} / \mathrm{L}$ 磷酸盐缓 冲液配制, $\mathrm{pH} 7.4), 4^{\circ} \mathrm{C}$ 放置 $10 \mathrm{~h}$. 经干冰一乙醇预冷的已烷速冻后, 放人 $-20^{\circ} \mathrm{C}$ 低温冰箱平 復, 恒冷箱切片, 片厚 $8 \mu \mathrm{m}$. 按 Sternberger 过氧化物酶-抗过氧化物酶法 (PAP 法) 以进行 5-HT 免疫细胞化学染色. 切片经 $0.05 \%$ 二氨基联苯胺和 $0.01 \% \mathrm{H}_{2} \mathrm{O}_{2}(0.05 \mathrm{~mol} / \mathrm{L} \mathrm{Tris}-\mathrm{HCl}$ 缓冲液配制, $\mathrm{pH} 7.6$ ) 显色后, $0.01 \mathrm{~mol} / \mathrm{L}$ 磷酸盐缓冲液漂洗 2 遍, 贴片于涂过 $1 \%$ 明胶的 载片上, 冷风吹干. 切片经冰浴 $50 \mathrm{mmol} / \mathrm{L}$ Tris $-\mathrm{HCl}$ 缓冲液冲洗 2 次, 每次 $5 \mathrm{~min}$, 然后进行 配体育. $2 \mathrm{nmol} / \mathrm{L}\left[{ }^{3} \mathrm{H}\right]$ 埃托啡 $\left(\left[{ }^{3} \mathrm{H}\right]-\mathrm{Et}\right) 25^{\circ} \mathrm{C} 30 \mathrm{~min}$, 冰浴 $50 \mathrm{mmol} / \mathrm{L}$ Tris $-\mathrm{HCl}$ 缓 冲液漂洗 2 次, 每次 $5 \mathrm{~min}$, 冰浴双蒸水略洗, 冷风吹干. 相邻切片用 $2 \mathrm{nmol} / \mathrm{L}\left[{ }^{3} \mathrm{H}\right]-\mathrm{Et}+$ $20 \mu \mathrm{mol} / \mathrm{L}$ 纳洛酮躬育以检查非特异结合, 部分切片用 $50 \mathrm{mmol} / \mathrm{L}$ Tris $-\mathrm{HCl}$ 缓冲液代替配 体捊育液以检查有无正化学显影. 用浸膜法 ${ }^{[6}$ 将空白盖玻片 $(25 \times 75 \mathrm{~mm}$, 上海工业玻璃四 厂, 酸及酒精清洗)涂一层核乳胶 (双蒸馏水 $1: 1$ 稀释), 置 5-10 ${ }^{\circ} \mathrm{C}$ 环境中晾干. 将涂有核乳 胶的盖玻片覆盖在贴有标本的载玻片上, 一端用粘合剂 (SA202, 上海合成树脂研 究 所) 粘 牢. 盖玻片上面再覆盖一张载玻片, 然后用橡皮筋捆牢. 黑纸包裹放人装有干燥剂的密封袋 中. 取几张涂有核乳胶的盖玻片, 经日光均匀曝光数秒钟后, 其中一部分立即按下述方法显 影, 其余部分用黑纸包好, 同时放人标本曝光密封袋内, 以检查有无负化学显影.标本在 5-10

本文 1990 年10月15日收到. 1991 年1月14日收到修改程. 
“C环境下曝光 6 周左右, D-19b 愿影 $3 \mathrm{~min}, \mathrm{~F}-5$ 定影 $10 \mathrm{~min}$ 左右,流水冲洗 $30 \mathrm{~min}$.

实验所用的药品: 5-HT 抗血清为美国 INCSTAR 公司产品; 羊抗兔 IgG 血清(效价 1:64), PAP 复合物 (效价 1:10) 为北京生物制品研究所产品; 核-4 型核乳胶为北京原子能科 学研究院产品; $\left[{ }^{3} \mathrm{H}\right]$ 埃托啡为上海医科大学放射药学教研室产品, 比活度为 $1702 \mathrm{~GB} / \mathrm{mmol}$; 盐酸纳洛酮为美国 Sigma 公司产品.

\section{二、结 果}

在 PAG 腹侧区中琏背核 5-HT 能神经元胞体及未梢分布的区域内有密集的银粒(图 1 (a)). 㲗育液中含 $20 \mu \mathrm{mol} / \mathrm{L}$ 纳洛酮时, 则银 粒数量明显减少(图1(b)). 未发现正负化学 显影.由此可见,中㖓背核及 PAG 腹侧区有 较密集的阿片受体, 在经过 $1 \%$ 多聚甲醛固 定及免疫细胞化学反应后, 仍保持其与配基 特异结合的能力.

在中缝背核区域内, 阿片受体有明显地 聚集于胞体的现象, 有时银粒排列成束状与 胞体部位相连. 图 2 显示中琏背核腹内侧部 5-HT 能神经元与阿片受体的关系. 聚集于 胞体的银粒并未均匀覆盖胞体, 而是多僱于 胞体一侧. 有时可见两个 5-HT 阳性神经 元,胞体均有银粒聚集,两胞体间有排列成束 的银粒连接. 有些 5-HT 阳性神经元胞体没

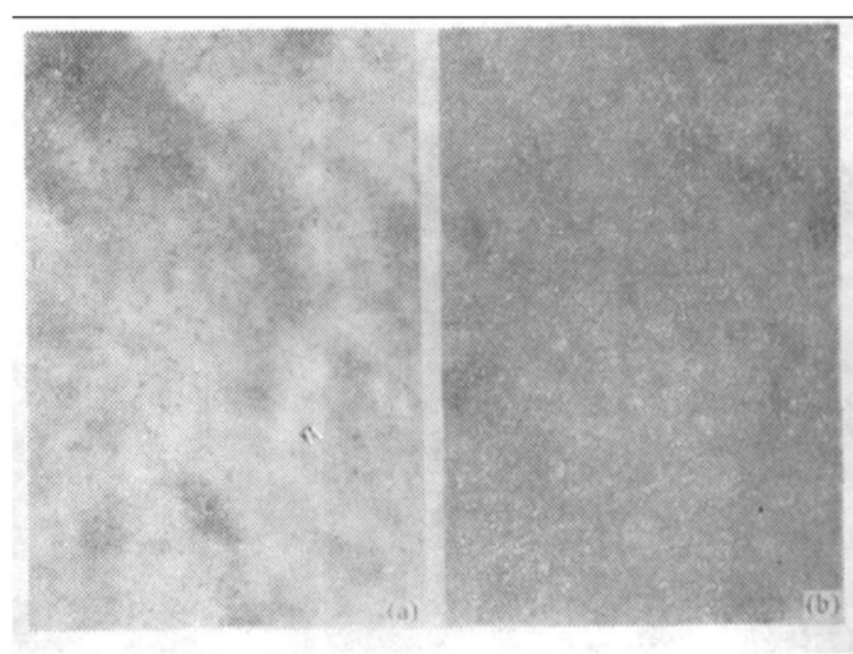

图 1

(a) PAG 腹侧区中㖓背核区域内密集的显影银柆; (b) 脬育液中含 $20 \mu \mathrm{mol} / \mathrm{L}$ 纳洛网时, 银粒数量明显 堿少 $(\times 240)$ 有银粒聚集的现象, 其表面覆盖的银粒并不比周围区域银粒密度高.

银粒聚集于胞体的现象并不限于中㖓背核。在脑桥网状核, 5-HT 阳性细胞散在, 显影银 粒也少, 但仍可见到银粒相对聚集于胞体的现象, 并且也有排列成束状的银粒与胞体部位相

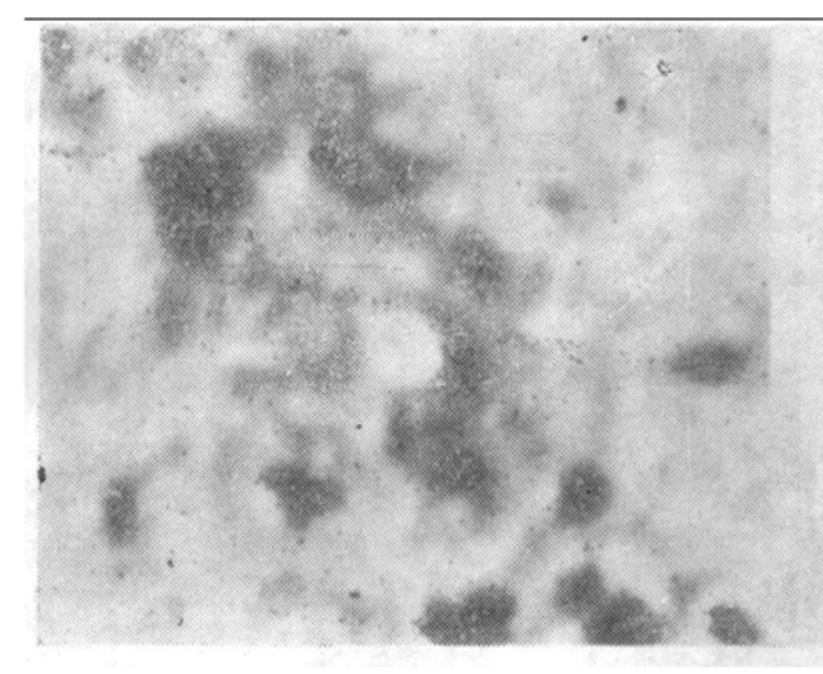

图 2 中琏背核腹内侧部 5-HT 阳性 神经元与阿片受体的关系 银粒相对聚集于盷体,有些银粒排列成束 状由胞体向外延伸相当距离 $(\times 240)$
图 3 脑桥网状核的两个 5-HT 阳性神经元

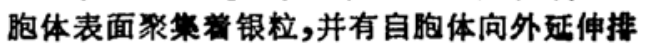
列成束的银柆 $(\times 240)$ 
连. 图 3 为脑桥网状核的两个 5-HT 能神经元。

\section{三、讨 论}

目前形态学研究技术发展的趋势是不同方法的联合使用. 已有人尝试用新鲜组织切片进

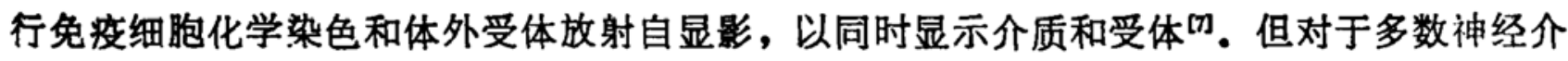
质来说, 免疫细胞化学染色在新鲜组织切片上不能满意地显示或根本无法加以显示. 我们在 前一段工作中, 用放射受体分析及受体放射自显影方法证明 $1 \%$ 多聚甲醴固定对受体结合能 力无明显影响 ${ }^{1}$. 在本工作中, 我们应用甲醴固定组织进行免疫细胞化学和体外受体放射自显 影双重显示,在国内外淌末见报道. 我们的结果初步表明, PAG 腹侧中缝背核区域存在着较 密集的阿片受体,在有些区域内阿片受体聚集于 5-HT 阳性神经元胞体,有些阿片受体排列成 与胞体部位相连的東,它们可能是轴突上的阿片受体.

上述结果对于阐明中枢微量注射药理实验中吗啡与 5-HT 能神经元相互作用的形态 基 础,提供了一些证据. 如果聚集于胞体的阿片受体确实位于 5-HT 能神经元上, 则吗啡可能 直接兴奋 5-HT 能神经元. 如果这些阿片受体位于其它神经元发出的支配 5-HT 能神经元的 轴突末梢上,则吗啡可能通过突触前抑制使 5-HT 能神经元脱抑制而兴奋. 想确定地得出结 论，还需要对阿片受体和 5-HT 能神经元的形态定位关系做电镜水平的精细分析.

致谢: 本文得到了刘鼎新和吕证宝教授的热心指导,在此谨致谢意.

\section{考文献}

[1] Steinbusch, H. V. M., in Handbook of Chemical Newroanatomy, 3(Eds. Bjorklund, A. et al.), Elserier, $1984,68-125$.

[2] Wamsley, J. K. et al., Newroscience, 7 (1983), 595-613.

[3] Oliverss, J. L. et al., Exp. Brain Res., 20 (1974), 32-44.

[4] Han, J. S. \& Xuan, Y. T., Intn. J. Neurosci., 29(1985), 109-118.

[5] Sternberger, L. A., Immunocytochemistry, 2nd ed., John Wiley and Sons, 1979.

[6]刘邪新,放射自显影技术,科学出版社, 1986,92-97.

[7] Ariano, M. A. \& Kenny, S. L., J. Neurosci. Mezhods, 15 (1985), 49-61.

1）马青平，榑士学位论文，特发表. 